\title{
Does the Self-Trapped Magnetic Polaron Exist in Electron-Doped Manganites?
}

\author{
H. Meskine, T. Saha-Dasgupta, ${ }^{*}$ and S. Satpathy \\ Department of Physics \& Astronomy, University of Missouri, Columbia, Missouri 65211, USA
}

(Received 9 April 2003; published 5 February 2004)

\begin{abstract}
We show from ab initio density-functional calculations and model studies that, in the electron-doped manganite $\mathrm{La}_{x} \mathrm{Ca}_{1-x} \mathrm{MnO}_{3}(x \ll 1)$, unbound electrons are introduced into the conduction band, which then trap themselves in the exchange-induced magnetic potential wells forming the self-trapped magnetic polarons (STMP). Hopping beyond the nearest neighbors drastically reduces the binding energy, while the Jahn-Teller coupling increases it somewhat, resulting in a net binding of about $100 \pm$ $20 \mathrm{meV}$. The electron is self-trapped in a seven-site ferromagnetic region, beyond which the lattice is essentially antiferromagnetic. In light of the recent experiments of Neumeier and Cohn, our results suggest that the STMP may be present in the lightly electron-doped manganites.
\end{abstract}

The bound magnetic polaron (BMP) observed in the magnetic semiconductors such as $\mathrm{Cd}_{1-x} \mathrm{Mn}_{x} \mathrm{Se}$ [1] consists of an electron bound to an impurity center, with the electron distorting the moments of the neighboring magnetic ions via exchange interaction. In contrast, the existence of the simpler and perhaps the more elegant counterpart, viz. the self-trapped magnetic polaron (STMP) [2-4], has not been conclusively established. In this case, the electron is bound, not by the impurity potential, but rather by the magnetic potential well, exchange induced by the electron itself (Fig. 1). Even though several materials, notably EuSe and EuTe, have been studied in the past as prime candidates for the STMP, it continues to remain an elusive entity [5-8].

In light of this, the recent experiments by Neumeier and Cohn [9], suggesting the existence of the magnetic polarons in the lightly electron-doped $\mathrm{CaMnO}_{3}$, are of considerable interest. These systems do satisfy several criteria conducive to the formation of the STMP, viz. (i) antiferromagnetic (AF) insulator with narrow $d$ band conduction states, (ii) strong exchange interaction between conduction electrons and core spins (via the Anderson-Hasegawa double exchange [10]), (iii) a moderately strong Jahn-Teller (JT) electron-lattice coupling, and (iv) the possible presence of unbound electrons introduced by La doping.

On the other hand, the formation of the STMP in the electron-doped $\mathrm{CaMnO}_{3}$ is contrary to the idea of de Gennes that doped electrons in the manganites are delocalized over the entire lattice producing a canted magnetic state [2]. However, the canted state is now well known to be unstable with respect to phase separation [11] or by correlation effects [12]. Also, using a onedimensional model, Batista et al. [13] have argued that in the limit of low doping the system is inhomogeneous, containing magnetic polarons, again in disagreement with the de Gennes result. Recently, Chen and Allen [14] developed a theoretical model for the STMP in $\mathrm{CaMnO}_{3}$. However, it lacked a realistic treatment of the energetics and also neglected electron hopping beyond the first nearest neighbor (1NN), which turns out to be an important destabilizing factor.

Model Hamiltonian and the Mott polaron. - The basic physics of the formation of the STMP is contained in the Hamiltonian, describing the double-exchange interaction between the $\operatorname{Mn}\left(e_{g}\right)$ electrons and the $\operatorname{Mn}\left(t_{2 g}\right)$ core spins:

$$
\mathcal{H}_{e l}=\sum_{i j}^{\prime} \sum_{a b}\left(t_{i j}^{a b} c_{i a}^{\dagger} c_{j b} \cos \frac{\theta_{i j}}{2}+\text { H.c. }\right)+\sum_{\langle i j\rangle} J S^{2} \cos \theta_{i j},
$$

where $c_{i a}^{\dagger}$ creates an electron at site $i$ with orbital index $a$ $\left(z^{2}-1\right.$ or $\left.x^{2}-y^{2}\right), \theta_{i j}$ is the angle between the (classical) $\operatorname{Mn}\left(t_{2 g}\right)$ core spins (denoted by $S$ ), arranged on a cubic lattice for $\mathrm{CaMnO}_{3}, J$ denotes the AF superexchange, $t_{i j}^{a b}$ is the hopping integral, and prime over the summation indicates sum over distinct bonds. The celebrated Anderson-Hasegawa double exchange [10] is contained in the $\cos (\theta / 2)$ dependence, which assumes an infinite Hund's rule penalty, so that the conduction electron spin is always aligned along the core spin.

A crude way to see the competition between the hopping term, which favors a ferromagnetic (FM) region of core spins in the vicinity of the electron, and the superexchange, which favors the AF core spins, is to consider the limit of a large polaron à la Mott [15]. Mott wrote the polaron energy as a sum of the confinement energy of the electron plus the superexchange cost,



FIG. 1. Sketch of a self-trapped magnetic polaron, showing the ferromagnetic region of core spins, exchange induced by the electron and in which the electron becomes self-trapped. 


$$
E_{\mathrm{Mott}}=\hbar^{2} \pi^{2} /\left(2 m R^{2}\right)+\nu J S^{2} \times\left(4 \pi R^{3} / 3\right),
$$

where $\nu$ is the number of NNs, $2 J S^{2}$ is the energy cost for forming the "wrong" FM bond, and the hopping parameters are contained in the effective mass $m$. The minimum of $E_{\text {Mott }}$ determines the radius $R$ and the energy of the polaron.

Jahn-Teller coupling. - Another relevant effect on top of this is the coupling to the lattice, dominated by the JT interaction between the doped electron and the on-site vibrational modes of the $\mathrm{MnO}_{6}$ octahedra [16-19]. The Hamiltonian describing it is

$$
\mathcal{H}_{\mathrm{JT}}=\sum_{i} \frac{1}{2} K Q_{i}^{2}-g \sum_{i, a b} c_{i a}^{\dagger}\left(Q_{i 2} \sigma_{x}^{a b}+Q_{i 3} \sigma_{z}^{a b}\right) c_{i b},
$$

where $Q_{i}^{2}=Q_{i 1}^{2}+Q_{i 2}^{2}+Q_{i 3}^{2}$, the three active octahedral distortion modes are the breathing mode $\left(Q_{1}\right)$, the inplane distortion mode $\left(Q_{2}\right)$, and the octahedral stretching mode $\left(Q_{3}\right), K$ is the elastic energy, $g$ is the JT coupling strength, and $\vec{\sigma}$ is the pseudospin describing the two $e_{g}$ orbitals, $|\uparrow\rangle=\left|z^{2}-1\right\rangle$ and $|\downarrow\rangle=\left|x^{2}-y^{2}\right\rangle$.

To see the effect of the JT interaction, we consider the energetics in the spirit of the Mott polaron. For the isolated $\mathrm{MnO}_{6}$ octahedron, the result is well known. The electron occupies the sole Mn site, producing an octahedral distortion that splits the double-degenerate $e_{g}$ level. The electronic energy gain is balanced by the elastic energy of the lattice, with a net energy gain of $-g^{2} / 2 K$ for the system [16]. In the solid, the electronic wave function is no longer localized at a single Mn site, but spreads to the adjacent sites as well. If we make a continuum approximation (large polaron limit) to describe this spreading, the extra energy gain on top of the Mott polaron energy, Eq. (2), is

$$
E_{\mathrm{JT}}=\int_{0}^{R}\left(-\frac{g^{2}}{2 K} n^{2}(r)\right) \times\left(4 \pi r^{2} d r / a^{3}\right)=-\frac{g^{2}}{2 K} c_{0}\left(\frac{a}{R}\right)^{3} .
$$

Here the number of electrons, associated with each of the $\left(4 \pi r^{2} d r / a^{3}\right)$ JT centers in the spherical shell between $r$ and $r+d r$, is given by $n(r)=(4 \pi / 3)(a / 2)^{3} \times$ $|\psi(r)|^{2}$, where $a$ is the Mn-Mn distance, $\psi(r)$ is the standard particle-in-a-box wave function with box radius $R$, and $c_{0} \approx 0.67$. Typical parameters for the manganites are [19-22] $t_{1 \mathrm{NN}} \equiv-t_{d d \sigma}(1 \mathrm{NN})=0.5-0.75 \mathrm{eV}$, $t_{2 \mathrm{NN}} \equiv-t_{d d \sigma}(2 \mathrm{NN})=0.2-0.3 \mathrm{eV}, \quad J S^{2}=5 \mathrm{meV}, \quad g=$ $2 \mathrm{eV} / \AA$, and $K=10-20 \mathrm{eV} / \AA^{2}$. Equation (4) shows that the JT coupling provides a small extra binding of $\sim 10 \mathrm{meV}$, taking a typical value $R \sim 2 a$.

Solution of the model Hamiltonian. - We have obtained the exact ground state of the magnetic polaron, which obviously goes beyond the simple Mott picture, for the model Hamiltonian,

$$
\mathcal{H}=\mathcal{H}_{e l}+\mathcal{H}_{\mathrm{JT}},
$$

by considering the most general wave function, $|\Psi\rangle=$ $\left|\psi_{e}\right\rangle \otimes|Q\rangle \otimes|\Theta\rangle$, where $\left|\psi_{e}\right\rangle=\sum_{i a} \psi_{i a} c_{i a}^{\dagger}|0\rangle$ is the electronic wave function, and $|Q\rangle=\Pi_{i}\left|Q_{2 i}, Q_{3 i}\right\rangle$ and $|\Theta\rangle=$ $\Pi_{i}\left|\theta_{i}\right\rangle$ are, respectively, the lattice and the core spin configurations. The total energy

$$
E=\langle\Psi|\mathcal{H}| \Psi\rangle=\sum_{i j}^{\prime} \sum_{a b}\left(t_{i j}^{a b} \psi_{i a}^{*} \psi_{j b} \cos \frac{\theta_{i j}}{2}+\text { c.c. }\right)+\sum_{\langle i j\rangle} J S^{2}\left(1+\cos \theta_{i j}\right)+\sum_{i} \frac{1}{2} K Q_{i}^{2}-g \sum_{i a b}\left(Q_{2 i} \sigma_{x}^{a b}+Q_{3 i} \sigma_{z}^{a b}\right) \psi_{i a}^{*} \psi_{i b}
$$

was then minimized subject to the normalization constraint for $\psi$, enforced by the Lagrange multiplier $\lambda$. Here $\theta_{i j}$ is the angle difference between the core spins at sites $i$ and $j$, and the zero of energy has been redefined by adding one in the superexchange term.

With the corresponding "free energy" $F=E+$ $\lambda\left(1-\sum_{i a}\left|\psi_{i a}\right|^{2}\right)$, an iterative procedure was followed to solve the set of equations, $\partial F / \partial \psi_{i a}=0, \partial F / \partial Q_{\alpha i}=$ $0(\alpha=2,3), \partial F / \partial \theta_{i}=0$, and $\partial F / \partial \lambda=0$. A large number of starting guess configurations were used in order to obtain the global energy minimum, which yields the polaron energy $E_{P}$. The polaron binding energy is the energy gain with respect to the energy of the electron in the AF lattice: $E_{B}=E_{\mathrm{AF}}-E_{P}$. Results presented here are for the cubic lattice with $L^{3}$ sites $(L=7)$ using free boundary conditions.

As already mentioned, the ground state is governed by a competition between the superexchange and the kinetic energy of the electron. As the $1 \mathrm{NN}$ hopping $t_{1 \mathrm{NN}}$ is increased, the polaron binding $E_{B}$ increases because of a larger kinetic energy gain by the spreading of the electron wave function. However, if hopping beyond the $1 \mathrm{NN}$ is allowed, then the situation changes fundamen- tally, since the electron can now hop (using $2 \mathrm{NN}$ hops along diagonal bonds) even in a completely AF lattice, thereby reducing the advantage of a local FM region. In fact, a sufficiently large $t_{2 \mathrm{NN}}$ destroys the magnetic polaron state favoring an $\mathrm{AF}$ lattice instead, so that $E_{B}$ goes to zero (Fig. 2).

We find that, for parameters relevant to the manganites, the ground state configuration of the core spins is either the AF lattice (if $t_{2 \mathrm{NN}}$ dominates over $t_{1 \mathrm{NN}}$ ) or a magnetic polaron with a seven-site FM region, although a 13-site FM region is sometimes favored (see Fig. 3). However, there is only a small difference in the energy between the latter two cases and the measured magnetic moment in the electron-doped $\mathrm{CaMnO}_{3}$ favors the seven-site polaron [9]. Both the electron wave function and the lattice distortions are strongly localized in the FM polaron region.

Density-functional studies. - As seen from Fig. 2, the binding energy is very sensitive to the model parameters, especially to $t_{2 \mathrm{NN}}$, with the polaron losing binding quickly around $t_{2 \mathrm{NN}} \sim 0.3 \mathrm{eV}$, which is also its typical value. In addition, other terms such as hopping to the oxygen atoms as well as to the upper Hund's-rule states 




FIG. 2. Binding energy of the magnetic polaron, showing how $t_{1 \mathrm{NN}}, t_{2 \mathrm{NN}}$, and $g$ affect it. The inset shows the groundstate energy for the AF lattice and for the seven-site FM polaron, as a function of the central-site distortion $Q_{2}$. Parameters for the inset are $t_{1 \mathrm{NN}}=0.75 \mathrm{eV}, t_{2 \mathrm{NN}}=0.3 \mathrm{eV}$, $g=2 \mathrm{eV} / \AA$, and $K=20 \mathrm{eV} / \AA^{2}$.

are missing in the model. To include these effects, we have performed a density-functional calculation of the STMP using a supercell technique and the linear-muffin-tinorbitals (LMTO-ASA) method [23].

Our supercell consisted of 32 formula units of $\mathrm{CaMnO}_{3}$, with one $\mathrm{Ca}$ replaced by a La, viz. $\left(\mathrm{La}_{1 / 32} \mathrm{Ca}_{31 / 32} \mathrm{MnO}_{3}\right)_{32}$, so that one electron per supercell is doped into the conduction bands of $\mathrm{CaMnO}_{3}$ (this amounts to a $\mathrm{La}$ doping of about 3\%). The generalized-gradient approximation within the local spin density-functional theory (DFT) was used for the exchange-correlation energy [24].

We performed two sets of calculations for the La-doped supercell. The first was for the type-G AF structure corresponding to bulk $\mathrm{CaMnO}_{3}$, which showed

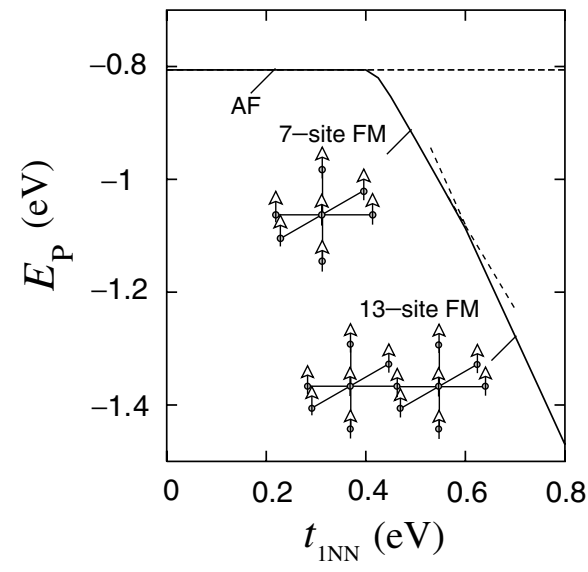

FIG. 3. Energy of the magnetic polaron as a function of the $1 \mathrm{NN}$ hopping. As $t_{1 \mathrm{NN}}$ is increased, the ground-state spin configuration changes from AF to the seven-site FM and finally to the 13-site FM core as indicated in the figure. Parameters used are $t_{2 \mathrm{NN}}=0.25 \mathrm{eV}, g=3 \mathrm{eV} / \AA$, and $K=10 \mathrm{eV} / \AA^{2}$. the doped electron to occupy the $\mathrm{CaMnO}_{3}$ conduction bands in a rigid-band fashion, confirming the presence of unbound electrons. There was no substantial change to the band structure with and without the La doping. Figure 4 shows the bands for the La-doped structure.

In the second calculation, the central $\operatorname{Mn}\left(t_{2 g}\right)$ spin was flipped, creating thereby the seven-site FM polaron cluster. The band structure is very similar to the first calculation, except that in the majority-spin channel two "magnetic polaron bands" occur in the gap, split off from the bottom of the conduction bands (Fig. 4). These two bands are derived from the two $\operatorname{Mn}\left(e_{g}\right)$ levels of the central Mn atom, with the wave function spread over the seven-site polaron region as seen from the charge-density contours (Fig. 5). A large Hund's-rule penalty $\left(J_{H} \approx\right.$ $3 \mathrm{eV}$ ) prevents any substantial leakage of the wave function into the surrounding AF region.

We use two ways to estimate the binding energy of the polaron: (i) from the position of the magnetic polaron bands (Fig. 4) and (ii) from total energy calculations. The first is given by the expression

$$
E_{B}^{\prime}=\Delta-1 / 2 K Q^{2}-2 \nu J S^{2},
$$

which consists of the one-particle energy gain $\Delta$, offset by the elastic energy cost and the loss of superexchange. Results are shown in Table I, with $\Delta$ estimated from Fig. $4, K \sim 10 \mathrm{eV} / \AA^{2}, J S^{2} \sim 5 \mathrm{meV}$, and $\nu=6$.

The second way is to simply take the total energy difference $E_{B}=E_{\mathrm{AF}}-E_{P}$, for which one should, in principle, optimize the structure (i.e., the JT distortions) for each of the two cases. To do this accurately, one has to use either the full-potential LMTO method or the linear

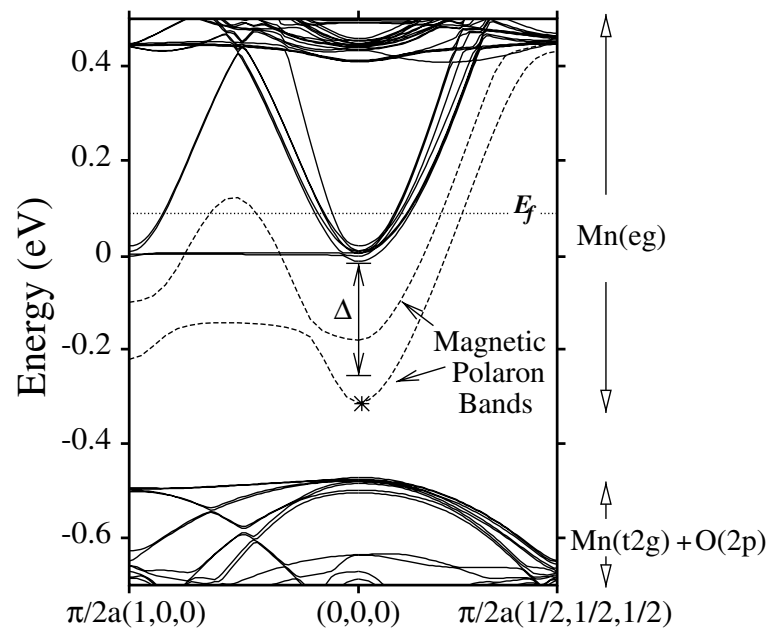

FIG. 4. Density-functional supercell band structure of $\left(\mathrm{La}_{1 / 32} \mathrm{Ca}_{31 / 32} \mathrm{MnO}_{3}\right)_{32}$ with AF type-G structure and lattice distortions $Q=0$ (solid lines). Majority and minority spin bands are identical. Dashed lines show, for the seven-site FM polaron case, the two "magnetic polaron" bands in the majority-spin channel that become introduced in the gap. $E_{F}$ shown is for the polaron case and there $Q_{2}=0.1 \AA$ for the central Mn site. 


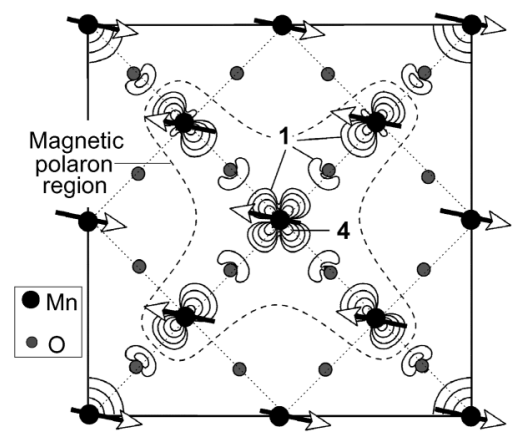

FIG. 5. Electronic charge-density of the STMP obtained from the DFT wave function at the $\Gamma$ point (indicated by an asterisk in Fig. 4). The electron is trapped in the seven-site polaron region (central-site plus the six $\mathrm{NN}$ sites). Contour values are $\rho_{n}=\rho_{0} \times 10^{n \delta}$, where $\rho_{0}=3.16 \times 10^{-5} e^{-} /(\text {Bohr radius })^{3}$, $\delta=0.5$, and $n$ labels the contours.

augmented-plane-wave method, both of which are intractable with the huge 160-atom supercell. However, since our model calculation (Fig. 2 inset) indicates that the polaron binding $E_{B}$ may be estimated using $Q=0$ (i.e., no structural optimization), a reasonable estimate of $E_{B}$ may be obtained from the simpler LMTO-ASA method. Thus, the atom positions in the two crystal structures were taken to be identical, the only difference between them being the flipping of the central-site $\operatorname{Mn}\left(t_{2 g}\right)$ spin.

In Table I, we present results, keeping just the $Q_{2}$ distortion at the central site in light of our model calculations that showed the rest of the distortions to be negligible. The estimated binding energy of the polaron is $100 \pm 20 \mathrm{meV}$, which is much weaker than the model results ( $\sim 400 \mathrm{meV}$ from Fig. 2, inset), but is consistent with the presence of additional hopping channels in the DFT calculations. The binding energy is robust enough that it is unlikely to be washed away by approximations such as the omission of quantum fluctuations of the core spins and the lattice.

The computed magnetic moment of the STMP is about $6.68 \mu_{B}$, as compared to $8.4 \pm 0.35 \mu_{B}$ obtained from magnetization measurements. For the ideal seven-site polaron, this number is expected to be $7 \mu_{B}, 6 \mu_{B}$ coming from the central $t_{2 g}$ spin changing from $S=-3 / 2$ to $+3 / 2$, and the remaining $1 \mu_{B}$ contributed from the alignment of the electron with the moment of the polaron region.

TABLE I. DFT results for the binding energy and net magnetic moment of the magnetic polaron in $\mathrm{La}_{x} \mathrm{Ca}_{1-x} \mathrm{MnO}_{3}$. Given are the central-site distortion $Q\left(Q_{2}=Q ; Q_{1}=Q_{3}=\right.$ $0), E_{B}^{\prime}$ computed from the one-particle energy gain $\Delta$ [Eq. (7)], and $E_{B}$ extracted from the DFT total energy differences. Errors in $E_{B}$ are due to the Brillouin zone integration errors.

\begin{tabular}{lccrc}
\hline \hline$Q(\AA)$ & $\Delta(\mathrm{meV})$ & $E_{B}^{\prime}(\mathrm{meV})$ & $E_{B}(\mathrm{meV})$ & $M$ \\
\hline $0 \AA$ & 180 & 120 & $90 \pm 10$ & $6.48 \mu_{B}$ \\
$0.1 \AA$ & 220 & 110 & $110 \pm 10$ & $6.68 \mu_{B}$ \\
\hline \hline
\end{tabular}

056401-4
Interestingly, conductivity experiments show an activated behavior with an activation energy in the range of 30-95 meV [9]. If we suppose the magnetic polarons to move by incoherent hopping, where the polaron hops via an intermediate state, then the activation energy would be expected to be a fraction of the binding energy, making the experimental result consistent with the calculated binding energy. However, this issue needs further study and awaits conductivity measurements on carefully prepared single crystal samples.

We acknowledge support of this work by the U.S. Department of Energy (DE-FG02-00ER45818).

*Permanent address: S. N. Bose National Center for Basic Sciences, JD Block, Salt Lake City, Kolkata 700098, India.

[1] For a review, see P. A. Wolff, Semiconductors and Semimetals, edited by J. K. Furdyna and J. Kossut (Academic, London, 1988), Vol. 25.

[2] P.-G. de Gennes, Phys. Rev. 118, 141 (1960).

[3] E. L. Nagaev, Sov. Phys. JETP Lett. 6, 18 (1967); ibid. 27, 122 (1968).

[4] E. L. Nagaev, Usp. Fiz. Nauk 117, 437 (1975) [Sov. Phys. Usp. 18, 836 (1976)].

[5] A. Mauger and D. L. Mills, Phys. Rev. B 31, 8024 (1985).

[6] M. Umehara, Phys. Rev. B 54, 5523 (1996).

[7] P. Wachter, Solid State Commun. 8, 473 (1970).

[8] T. Kasuya et al., Solid State Commun. 8, 1543 (1970).

[9] J. J. Neumeier and J. L. Cohn, Phys. Rev. B 61, 14319 (2000); J. L. Cohn and J. J. Neumeier, Phys. Rev. B 66, 100404 (2002).

[10] P.W. Anderson and H. Hasegawa, Phys. Rev. 100, 675 (1955).

[11] A. Moreo et al., Science 283, 2034 (1999).

[12] S. K. Mishra et al., Phys. Rev. B 55, 2725 (1997).

[13] C. D. Batista et al., Phys. Rev. B 58, R14 689 (1998).

[14] Y.-R. Chen and P. B. Allen, Phys. Rev. B 64, 064401 (2001).

[15] N. F. Mott, Metal-Insulator Transitions (Taylor \& Francis, London, 1974).

[16] See, e.g., I. B. Bersuker, The Jahn-Teller Effect and Vibronic Interactions in Modern Chemistry (Plenum, New York, 1984).

[17] J. H. Van Vleck, J. Chem. Phys. 7, 72 (1939).

[18] J. Kanamori, Jpn. J. Appl. Phys., Suppl. 31, 14 (1960).

[19] Z. S. Popović and S. Satpathy, Phys. Rev. Lett. 84, 1603 (2000); ibid. 88, 197201 (2002).

[20] S. Satpathy, Z. S. Popović, and F. R. Vukajlović, Phys. Rev. Lett. 76, 960 (1996); J. Appl. Phys. 79, 4555 (1996).

[21] T. Saha-Dasgupta and S. Satpathy, J. Phys. C 15, 1685 (2003).

[22] W. E. Pickett and D. J. Singh, Phys. Rev. B 53, 1146 (1996).

[23] O. K. Andersen, Phys. Rev. B 12, 3060 (1975); O. K. Andersen and O. Jepsen, Phys. Rev. Lett. 53, 2571 (1984).

[24] J. P. Perdew and Y. Wang, Phys. Rev. B 45, 13244 (1992); J. P. Perdew, K. Burke, and M. Ernzerhof, Phys. Rev. Lett. 77, 3865 (1996). 